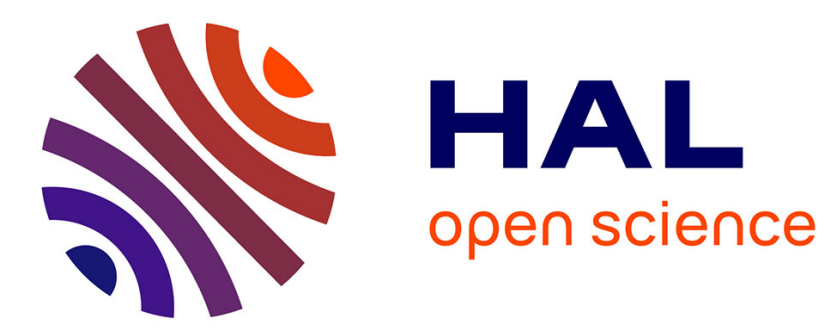

\title{
Simulation of a hot coaxial jet: direct noise prediction and flow-acoustics correlations
}

\author{
Christophe Bogey, Sébastien Barré, Daniel Juvé, Christophe Bailly
}

\section{To cite this version:}

Christophe Bogey, Sébastien Barré, Daniel Juvé, Christophe Bailly. Simulation of a hot coaxial jet: direct noise prediction and flow-acoustics correlations. Physics of Fluids, 2009, 21, pp.035105. 10.1063/1.3081561 . hal-00461424

\section{HAL Id: hal-00461424 \\ https://hal.science/hal-00461424}

Submitted on 14 Jun 2012

HAL is a multi-disciplinary open access archive for the deposit and dissemination of scientific research documents, whether they are published or not. The documents may come from teaching and research institutions in France or abroad, or from public or private research centers.
L'archive ouverte pluridisciplinaire HAL, est destinée au dépôt et à la diffusion de documents scientifiques de niveau recherche, publiés ou non, émanant des établissements d'enseignement et de recherche français ou étrangers, des laboratoires publics ou privés. 


\title{
Simulation of a hot coaxial jet: Direct noise prediction and flow-acoustics correlations
}

\author{
Christophe Bogey, ${ }^{a)}$ Sébastien Barré, ${ }^{\text {b) }}$ Daniel Juvé, ${ }^{\mathrm{c})}$ and Christophe Bailly ${ }^{\mathrm{d})}$ \\ Laboratoire de Mécanique des Fluides et d'Acoustique, UMR CNRS 5509, Ecole Centrale de Lyon, \\ 69134 Ecully Cedex, France
}

(Received 3 October 2008; accepted 8 January 2009; published online 13 March 2009)

\begin{abstract}
A coaxial jet originating from parallel coplanar pipe nozzles is computed by a compressible large eddy simulation (LES) using low-dissipation and low-dispersion schemes in order to determine its acoustic field and to study noise generation mechanisms. The jet streams are at high velocities, the primary stream is heated, and the Reynolds number based on the primary velocity and the secondary diameter is around $10^{6}$. High levels of turbulence intensity are also specified at the nozzle exit. The jet aerodynamic field and the near-pressure field are both obtained directly from the LES. The far-field noise is calculated by solving the linear acoustic equations, from the unsteady LES data on a cylindrical surface surrounding the jet. A good agreement is observed in terms of directivity, levels, and narrow-band spectra with noise measurements carried out during the EU project CoJeN for a coaxial jet displaying same stream velocities and temperatures, coplanar nozzle outlets with identical area ratio, and a high Reynolds number. However, certainly due to differences in the properties of the nozzle-exit boundary layers with respect to the experiment, some unexpected peaks are noticed in the simulation spectra. They are attributed to the development of a Von Kármán street in the inner mixing layer and to vortex pairings in the outer shear layer. High correlation levels are also calculated between the pressure waves radiated in the downstream direction and flow quantities such as axial velocity, vorticity norm, density, and temperature, taken around the end of the primary and secondary potential cores. Noise generation in the coaxial jet therefore appears significant around the end of the two potential cores. These flow regions are characterized by intermittency, a dominant Strouhal number, and variations in the convection velocity as similarly found in single jets. The use of density or temperature to compute flow-noise correlations finally seems appropriate for a heated jet flow, but might lead to correlations with acoustic disturbances in the potential core. () 2009 American Institute of Physics. [DOI: 10.1063/1.3081561]
\end{abstract}

\section{INTRODUCTION}

Jet noise simulations developed actively during the last decade, with the aim of providing a better understanding of sound generation, notably by complementing current experimental and theoretical findings, ${ }^{1,2}$ as well as reliable predictions for practical flows. The rapid advances in this area have been summarized by Colonius and Lele ${ }^{3}$ and by Wang et al. ${ }^{4}$ The feasibility of computing jet noise directly by solving the unsteady compressible Navier-Stokes equations has been demonstrated for simple configurations. Subsonic single unheated jets at low and moderate Reynolds numbers have thus been calculated by direct numerical simulations and large eddy simulations (LES) ${ }^{5-7}$ These preliminary simulations also allowed us to investigate noise generation mechanisms under controlled conditions. Recent works of the present authors ${ }^{8,9}$ were, in particular, focused on the properties of the

\footnotetext{
${ }^{a}$ CNRS Research Scientist. Telephone: 334721860 18. Fax: 334721891 43. Electronic mail: christophe.bogey@ec-lyon.fr.

${ }^{b)}$ Electronic mail: sebastien.barre@dassault-aviation.com. Present address: Dassault Aviation, 78 quai Marcel Dassault, Cedex 300, 92552 Saint Cloud Cedex, France.

${ }^{c}$ Professor at Ecole Centrale de Lyon. Electronic mail: daniel.juve@eclyon.fr.

d) Professor at Ecole Centrale de Lyon and at Institut Universitaire de France, Electronic mail: christophe.bailly@ec-lyon.fr.
}

two components in subsonic jet noise as the jet Mach and Reynolds numbers vary and on the correlations between jet turbulence and radiated pressure field.

In order to get closer to realistic jets, there is a need for simulations of more complex configurations, which may be characterized by high Reynolds numbers, high temperatures, and coaxial geometries, among other parameters. With this aim in view, the effects of the presence of the nozzle lips in the computational domain have, for instance, been studied for subsonic jets at high Reynolds numbers by Bogey et al. ${ }^{10}$ and Uzun and Hussaini. ${ }^{11}$ Screech noise generation has been studied numerically by Al-Qadi and Scott, ${ }^{12}$ Berland et al.,${ }^{13}$ and $\mathrm{Li}$ and $\mathrm{Gao}^{14}$ in rectangular, planar, and round underexpanded supersonic jets. Simulations of the sound field obtained from beveled jet nozzles have been also performed by Paliath et al. ${ }^{15}$ and Viswanathan et al. ${ }^{16}$ Finally predictions of the noise generated by dual-stream jets have been carried out using LES by Andersson et al. ${ }^{17}$ Vuillemin et al. ${ }^{18}$ Mihăescu et al. ${ }^{19}$ Viswanathan et al. ${ }^{16}$ Tristanto et al. ${ }^{20}$ and Eschricht $e t$ al. ${ }^{21}$

Dual-stream nozzles are of interest for the aerospace industry. However, knowledge of aerodynamic development and noise generation in these flow configurations still requires extensive research efforts. Several parameters such as the stream temperatures, the nozzle geometry, the velocity 
ratio, and the inflow conditions are indeed expected to have significant effects on physical mechanisms taking place within the jets. The primary stream of coaxial jets is, for instance, usually strongly heated, which might affect noise generation according to the controversial debate about the presence of additional dipolar sound sources in hot jets, which is mentioned in Tanna ${ }^{22}$ and Viswanathan. ${ }^{23}$ Coaxial jets also exhibit complicated flow structures, depending on the mean-velocity ratio between the two streams. ${ }^{24,25}$ Measurements of $\mathrm{Ko}$ and $\mathrm{Kwan}^{24}$ within the initial region of coaxial jets isolated, for example, three mixing regions, which are as many noise generation regions. These authors then proposed to consider coaxial jets as combinations of several single jets, which was later used by Fisher et al. ${ }^{26,27}$ to formulate basic noise models. More recently Tinney and Jordan $^{28}$ analyzed measurements performed in the nearpressure fields of a number of coaxial jets and observed two distinct acoustic features as in single jets. The dynamics of the jet responsible for each of these signatures remain, however, poorly understood.

In the present work, a coaxial jet is computed by a compressible LES using low-dissipation and low-dispersion schemes. ${ }^{29}$ The near-pressure field is determined directly by LES and extrapolated in far field by solving the linear acoustic equations using LES data recorded on a cylindrical surface surrounding the jet. The nozzle consists, for simplicity, of parallel pipes, and the nozzle lips are coplanar. The jet streams are at conditions specified in the EU project CoJeN. ${ }^{30-32}$ The primary and secondary streams are defined by velocities $U_{p}=404.5 \mathrm{~m} / \mathrm{s}$ and $U_{s}=306.8 \mathrm{~m} / \mathrm{s}$ and by static temperatures $T_{p}=775.6 \mathrm{~K}$ and $T_{s}=288.1 \mathrm{~K}$. The ratio between the nozzle diameters is $D_{s} / D_{p}=2$. The secondary diameter of the computed jet is $D_{s}=4.9 \mathrm{~cm}$, which leads to the Reynolds number $\operatorname{Re}=U_{p} D_{s} / \nu=1.3 \times 10^{6}$. For LES concerns, this value is lower than the Reynolds number of the corresponding CoJeN jet with $D_{s}=20 \mathrm{~cm}$. The first objective of the present study is to compute the turbulent development and the pressure field of the coaxial jet by LES and to assess the accuracy of direct noise prediction for this complex flow configuration in terms of directivity, levels, and narrow-band spectra. The features of the far acoustic field estimated by the simulation are therefore compared with CoJeN measurements, although some discrepancies are expected due to differences in the properties of the nozzle-exit boundary layers between computation and experiment. In order to identify possible regions of noise generation in the coaxial jet, correlations are then computed between the jet flow field and the pressure acoustic field, as it has been previously done in single-stream jets both from experimental ${ }^{33,34}$ and numerical ${ }^{9}$ database. Considering the results found for single jets, we focus on the flow features along the primary and secondary potential core axes, located, respectively, at $r=0$ and $r=0.375 D_{s}$, and on the pressure waves radiated in the downstream direction, in order to obtain significant correlation levels. The use of density or temperature to compute flownoise correlations instead of velocity or vorticity is moreover discussed.

This paper is organized as follows. The parameters of the LES of the coaxial jet and those of the extrapolation of the
LES near field in the acoustic far field, including in both cases numerical algorithm, computational grids, and times, are documented in Sec. II. The jet initial conditions at the exit of the coplanar nozzles are also presented in that section. The main aerodynamic results obtained for the jet are reported in Sec. III, where properties of the flow along the potential core axes as well as the lip lines are also described. Acoustic near-field and far-field results are then shown and discussed in Sec. IV. To evaluate the accuracy of noise prediction, they are compared with CoJeN data when possible. In Sec. V, correlations between turbulence and acoustics are presented with the aim of identifying possible sound sources. Concluding remarks are provided in Sec. VI. Finally note that the velocity of the primary jet stream $U_{p}$ and the diameter of the secondary nozzle $D_{s}$ are used throughout the paper to normalize velocities and distances, as agreed in the CoJeN project.

\section{COMPUTATIONAL PARAMETERS}

\section{A. Compressible LES of the jet}

The LES is performed using an in-house finite-difference solver of the three-dimensional cylindrical filtered compressible Navier-Stokes equations, developed for noise computations using centered ${ }^{29}$ and noncentered ${ }^{35}$ schemes with lowdissipation and low-dispersion properties. The axis singularity is taken into account by the method of Constantinescu and Lele ${ }^{36}$ based on series expansions. Fourth-order 11-point finite differences are used for spatial discretization, and a second-order six-stage low-storage Runge-Kutta algorithm is applied for time integration. To ensure numerical stability, grid-to-grid oscillations are removed every time step by an explicit 11-point filtering of the flow variables, which is optimized in the wave-number space to damp only the short waves discretized by fewer than four points per wavelength. The filtering enables also to take into account the effects of the subgrid energy-dissipating scales without affecting significantly the resolved scales, as described in detail in previous works. ${ }^{37}$ This LES approach was developed to preserve the Reynolds number given by the inflow conditions, which might not be possible using eddy-viscosity subgrid models such as the dynamical Smagorinsky model. ${ }^{38,39}$ Finally, in order to compute the radiated noise directly, nonreflective boundary conditions are implemented, with the addition of a sponge zone in the jet at the outflow. ${ }^{40}$

The LES is conducted on a computational domain of $14 \times 10^{6}$ points including two straight pipe nozzles with coplanar lips, which is presented in Fig. 1. The lip of the outer pipe has a thickness of $0.013 D_{s}$, whereas the inner lip is infinitively thin. Two overlapping cylindrical grids are used. The first grid of $n_{z} \times n_{r} \times n_{\phi}=391 \times 98 \times 48$ points is devoted to the interior of the pipes, from their inlet boundary at $z=-3.3 D_{s}$. The second grid of $n_{z} \times n_{r} \times n_{\phi}=867 \times 295 \times 48$ points contains the jet flow and the acoustic near field. It extends up to $z=18 D_{s}$ in the axial direction and to $r=8 D_{s}$ in the radial direction from the jet axis. The overlapping zone is from $z=-0.30 D_{s}$ to $z=-0.23 D_{s}$ and consists of the coinciding ten last rows of the pipe grid and ten first rows of the jet grid in the axial direction. In this direction, the grid spacing 

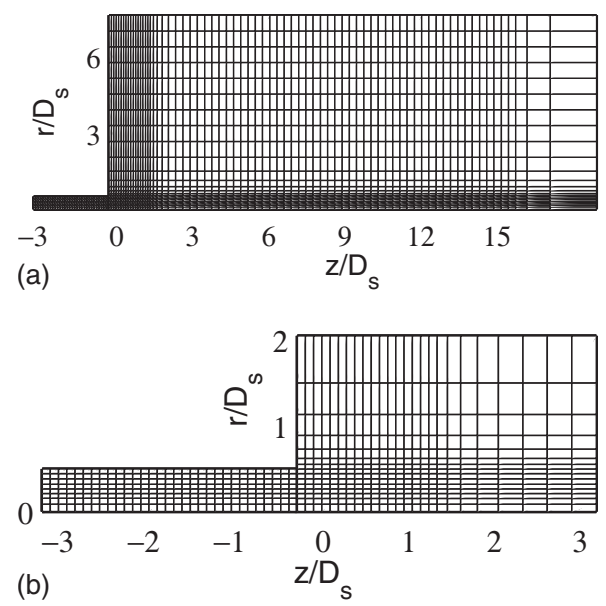

FIG. 1. Representation of the $(z, r)$ section of the LES mesh grid. Top: full computational domain; bottom: zoom in the pipe region. Every 12th grid point is shown in both axial and radial directions.

is $0.008 D_{s}$ in the pipe and up to $z=1.3 D_{s}$ outside the nozzle. A stretching rate of $2 \%$ is then applied, so that the axial grid spacing is $0.024 D_{s}$ for $z>2 D_{s}$. The grid is then uniform up to $z=15 D_{s}$, from which an $8 \%$ stretching is used to form the sponge zone. In the radial direction, the mesh spacing is minimum and equal to $0.0045 D_{s}$ at the nozzle walls. It increases outside of the jet flow at a rate of $3.5 \%$ to reach $0.052 D_{s}$ for $r>1.9 D_{s}$ from the jet axis. The sound field is therefore expected to be properly resolved up to Strouhal number $\mathrm{Sr}_{\max }=c_{\mathrm{amb}} D_{s} /\left(U_{p} \times 4 \times 0.052 D_{s}\right)=4$, where $c_{\mathrm{amb}}$ $=340.3 \mathrm{~m} / \mathrm{s}$ is the sound speed in the ambient medium, and the pressure in the ambient medium is $p_{\mathrm{amb}}=101325 \mathrm{~Pa}$.

The LES is carried out during 400000 time steps $\left(\Delta t_{\mathrm{LES}}=7.4 \times 10^{-8} \mathrm{~s}\right.$ or $\left.\Delta t_{\mathrm{LES}} U_{p} / D_{s}=6.1 \times 10^{-4}\right)$ and requires an amount of $1800 \mathrm{CPU}$ hours on a supercomputer Nec SX5. The length of the initial run, before initiating averaging, is 120000 time steps. The statistics of the flow are therefore calculated during 280000 time steps, which corresponds to the nondimensional averaging time of $\Delta T U_{p} / D_{s}$ $=170.7$. Time signals of velocity are also recorded in the jet over cylindrical surfaces located at $r=0, D_{s} / 4,3 D_{s} / 8$, and $D_{s} / 2$ from the centerline, every $50 \Delta t_{\mathrm{LES}}$ during the final 260000 LES iterations. For the computation of velocity spectra, they are divided into 25 overlapping sections of length $20000 \Delta t_{\text {LES }}$, windowed by a Hanning function.

\section{B. Jet initial parameters}

In order to define turbulence conditions at the nozzle exit as realistic as possible at the high Reynolds number of the present LES, small velocity disturbances random both in space and in time are added at the pipe inflow to seed the turbulence, in the same way as recently carried out by Bogey et $a l{ }^{10}$ for single-stream jets. The inflow perturbations are growing along the pipes to provide significant turbulence intensities at the nozzle exit. To illustrate the initial conditions thus obtained, the radial profiles of the mean axial velocity and of the root-mean-square levels of the fluctuating axial velocity at the nozzle exit are presented in Fig. 2. The nozzle-exit boundary layers exhibit thicknesses around
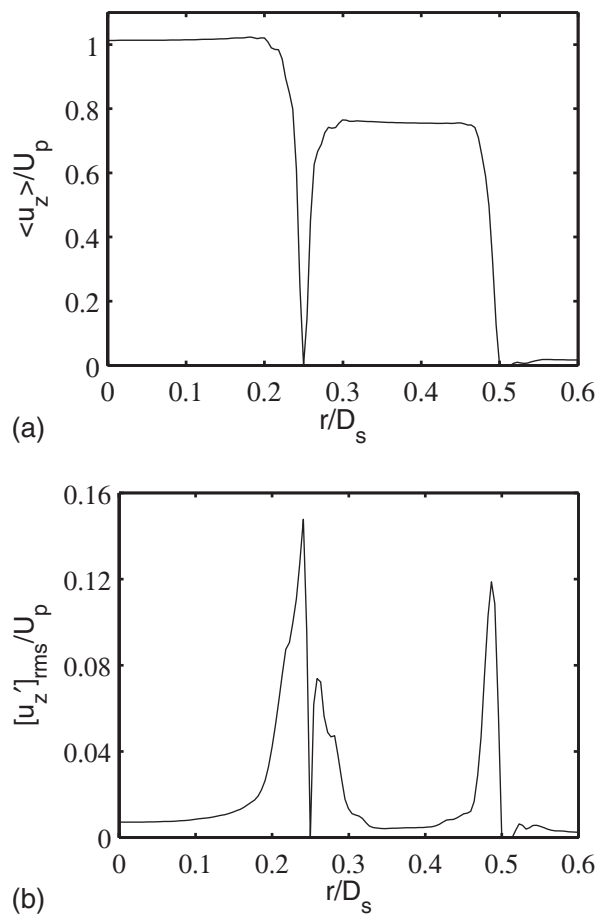

FIG. 2. Radial profiles at the exit of the coplanar nozzle at $z=0$ : (a) mean axial velocity, $\left\langle u_{z}\right\rangle / U_{p}$; (b) rms levels of the fluctuating axial velocity, $\left[u_{z}^{\prime}\right]_{\mathrm{rms}} / U_{p}$.

$0.032 D_{s}$ and peak levels of axial turbulence intensity $\left[u_{z}^{\prime}\right]_{\mathrm{rms}} / U_{p} \simeq 0.14,0.08$, and 0.12 , respectively, from the inner to the outer boundary layers.

When the present method is used in the LES for specifying the jet initial conditions at the nozzle exit, no forcing is applied directly within the jet flow. Previous jet simulations ${ }^{41}$ performed without nozzle in the computational domain demonstrated indeed that an intrusive forcing might have a strong influence on jet development and acoustic radiation. Moreover, using this approach, high levels of turbulence intensities can be obtained without generating significant spurious noise. This may enable to avoid the generation of an additional vortex-pairing noise which was observed in initially laminar jet flows. ${ }^{10,42,43}$ It must be, however, noted that, because of the numerical cost of the simulation and of the high Reynolds number of the jet, the boundary layers inside the pipe nozzles are unfortunately under-resolved.

In the present simulation, the inflow conditions are specified to mimic experimental conditions with as much fidelity as possible, but they might lead to discrepancies with respect to CoJeN measurements. Geometrical differences between computation and experiment can be first mentioned. The nozzle pipe in the LES is straight whereas a convergent nozzle is used in the experiment. In addition the inner lip is infinitively thin in the simulation, whereas the experimental inner lip is $0.005 D_{s}$ thick, and ends in a half-circle. As pointed out by Viswanathan and Clark, ${ }^{44}$ this will likely affect the initial development of the shear layers, and consequently the noise sources in these flow regions.

More generally, the parameters of the incoming boundary layers should be ideally the same in computation and experiment, which is not the case here because of the nu- 
merical constraints as well as of the lack of detailed information on the nozzle-exit boundary layers in the CoJeN experiment. The need for high initial turbulence intensities at a high Reynolds number has been emphasized above. One another important issue, noticed, in particular, by Bodony and Lele, ${ }^{45}$ is the thickness of the boundary layers. Zaman ${ }^{46}$ measured, for instance, initial momentum thicknesses around 0.003 diameter in single-stream jets at Reynolds numbers between $10^{5}$ and $2.5 \times 10^{5}$. The boundary-layer thickness of $0.032 D_{s}$ in the present simulated jet therefore appears quite large compared to what could be expected in the CoJeN jet, and one can expect the shear-layer phenomena to develop at downshifted frequencies. Thick boundary layers also usually result in short potential cores. ${ }^{10,45}$ However, the effects may be smaller on turbulence along the potential core axes than on turbulence along the shear layers. ${ }^{41}$ For these reasons, in order to avoid attributing physical interpretations to simulation artifacts, noise sources will be only investigated along the jet core axes in Sec. V.

\section{Extrapolation of the LES acoustic field}

For the characterization of the noise generated by the coaxial jet, and for the comparison with CoJeN experimental data, the acoustic near field obtained directly by LES is propagated in far field. Different extrapolation methods can be applied. They are based, for instance, on the integral solution of Kirchhoff equation ${ }^{47,48}$ or on the use of a set of acoustic equations. ${ }^{10,49}$ In the present work, the sound propagation is taken into account by solving the linear acoustic equations written for the unknown variables $\left(u_{z}^{\prime}, u_{r}^{\prime}, u_{\phi}^{\prime}, p^{\prime}\right)$, as in Berland et al., ${ }^{13}$ for a cylindrical geometry. The numerical methods used for this calculation are the same as those used for the LES. The noncentered 11-point finite differences and filters developed by Berland et al. $^{35}$ are, in particular, applied at the inner-side boundary where the LES data are introduced.

In practice, the LES velocity and pressure are recorded over an open cylindrical control surface located around the jet at $2 D_{s}$ from the jet axis, at every point between $0.3 D_{s}$ $\leq z \leq 13.8 D_{s}$, every $25 \Delta t_{\text {LES }}$ during the final $260000 \mathrm{LES}$ time steps. Note that the acoustic extrapolation has been also performed from control surfaces at $3 D_{s}$ and $4 D_{s}$ from the axis. Small effects on the far acoustic field have been found. ${ }^{50}$

The velocity and pressure fluctuations are introduced at the bottom boundary of a cylindrical grid of $n_{z} \times n_{r} \times n_{\phi}$ $=1771 \times 1201 \times 48$ points on which the linear acoustic equations are solved. From the control surface, the noise is propagated at a distance of $60 D_{s}$ from the nozzle exit as in CoJeN experiments, on the extrapolation grid presented in Fig. 3. The calculation requires 80 CPU hours using a Nec SX5.

In the axial direction, the discretization is that of the LES for $0 \leq z \leq 14 D_{s}$. A stretching rate of $4 \%$ is then applied to get mesh spacings of $0.05 D_{s}$ and $0.1 D_{s}$ in the downstream and upstream directions, respectively. In the radial direction, the grid is uniform with a mesh spacing of $0.05 D_{s}$. Therefore the sound field should be accurately calculated up to Strouhal number $\mathrm{Sr}_{\max }=c_{\mathrm{amb}} D_{s} /\left(U_{p} \times 4 \times 0.05 D_{s}\right)=4.2$, which is very

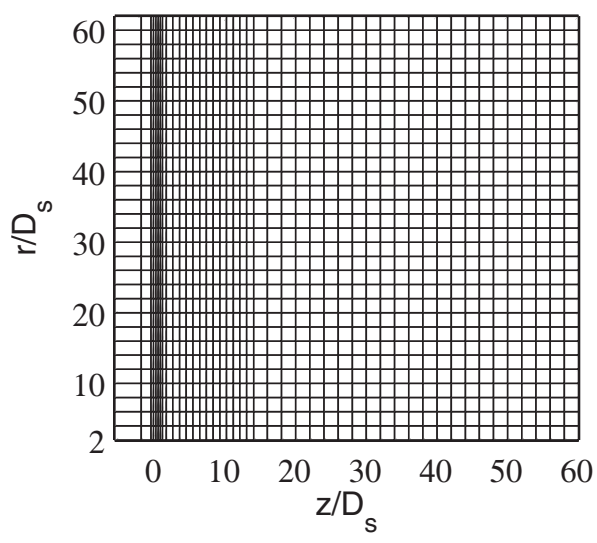

FIG. 3. Representation of the $(z, r)$ section of the mesh grid used for the far-field propagation from the surface at $r=2 D_{s}$. Every 40th grid point is shown in both axial and radial directions.

similar to the value $\mathrm{Sr}_{\max }=4$ obtained in the LES. The time step of the propagation computation, or acoustic time step, is $25 \Delta t_{\text {LES }}$. As a result, there is no spatial nor temporal interpolation of the LES near-field data. The extrapolation is performed during 14400 acoustic time steps. In order to study the radiated sound field, pressure signals are recorded around the jet at distances of $60 D_{s}$ from the nozzle exit, as it will be illustrated in Fig. 16, during 9200 acoustic time steps (or 230000 LES time steps or a nondimensional time $\left.\Delta T U_{p} / D_{s}=140.2\right)$. For the computation of pressure spectra, they are divided into 20 overlapping sections of length corresponding to 876 acoustic time steps, windowed by a Hanning function. The sound spectra are also averaged in the azimuthal direction, over the $48(z, r)$ sections of the computational grid.

\section{AERODYNAMIC RESULTS}

The main aerodynamic results obtained for the coaxial jet by LES are reported and compared with CoJeN preliminary particle image velocimetry (PIV) data. ${ }^{31,32}$ The development of turbulence along the lip lines, as well as along the potential core axes, is also investigated.

\section{A. Instantaneous flow fields}

Snapshots of the vorticity norm and of the static temperature downstream of the nozzle are represented in Fig. 4 for $z<8 D_{s}$. The development of the turbulence and the mixing of the cold and hot streams within the inner shear layer are clearly visible. The jet growth appears to take predominantly place in the outer shear layer, whereas a Von Kármán vortex street, which will be displayed more obviously in Fig. 9, is noted in the inner shear layer. The latter observation is probably due to the relatively small difference in velocity between the two streams, and it will be discussed later in Sec. III D. The inner and outer shear layers are found to interact around $z=2 D_{s}$ before the end of the primary potential core which can be roughly evaluated around $z=4 D_{s}$. Downstream, the flow appears turbulent, even if large spots of high temperature can still be noticed as it is the case in Fig. 4(b) at $z \simeq 5.5 D_{s}$. 

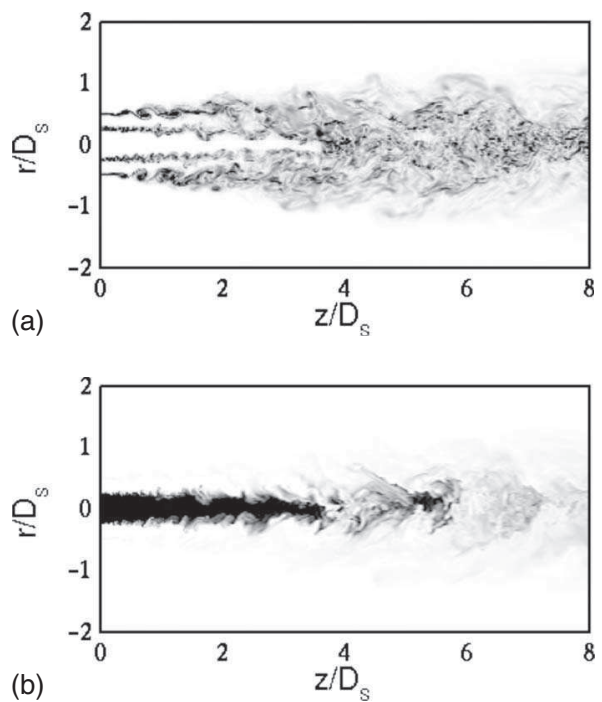

FIG. 4. Snapshots in the $(z, r)$ section: (a) vorticity norm $|\boldsymbol{\omega}|$, (b) static temperature $T$. The gray scales are for $0 \leq|\boldsymbol{\omega}| \times D_{s} / U_{p} \leq 12.1$ and for $T_{\mathrm{amb}} \leq T \leq T_{p}$, respectively, from light to dark.

\section{B. Mean flow fields}

To illustrate the development of the jet mean flow, contours of the mean axial velocity are shown in Fig. 5. As mentioned above, the primary potential core is longer than the secondary potential core. More precisely the lengths of the primary and the secondary cores, obtained here arbitrarily when $\left\langle u_{z}\right\rangle=0.95 U_{p}$ on the jet centerline and when $\left\langle u_{z}\right\rangle=0.95 U_{s}$ at $r=0.375 D_{s}$, are, respectively, $z_{c}=3.5 D_{s}$ and $z_{c}=1.5 D_{s}$.

As in most LES, the potential core of the simulated jet is shorter than that estimated in experiments. For the present coaxial jet, $z_{c}=5.4 D_{s}$ is, for example, obtained along the jet centerline by CoJeN partners using PIV measurements. In agreement with the discussion in Sec. II B, this discrepancy can be associated with differences in the initial development of the mixing layers due to the thick incoming boundary layers in the simulation.

Despite this, the velocity decay along the jet centerline is investigated by plotting in Fig. 6(a) the LES profile of mean axial velocity along $r=0$, as well as the corresponding CoJeN PIV profiles shifted by $-2 D_{s}$ in the $z$-direction for the comparison. The numerical and experimental velocity decays are found to be very similar. The profile of mean axial ve-

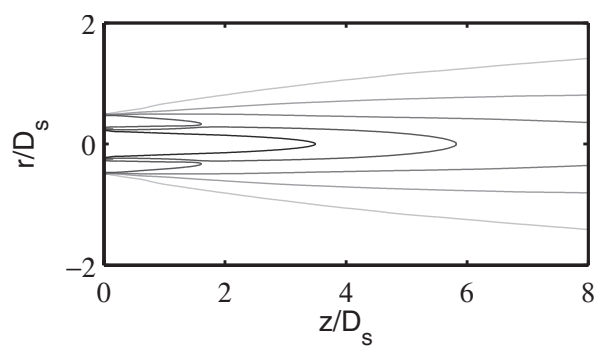

FIG. 5. Representation in the $(z, r)$ section: contours of mean axial velocity $\left\langle u_{z}\right\rangle / U_{p}=[0.03,0.26,0.49,0.72,0.95]$, with $0.72 U_{p}=0.95 U_{s}$, from gray to black.
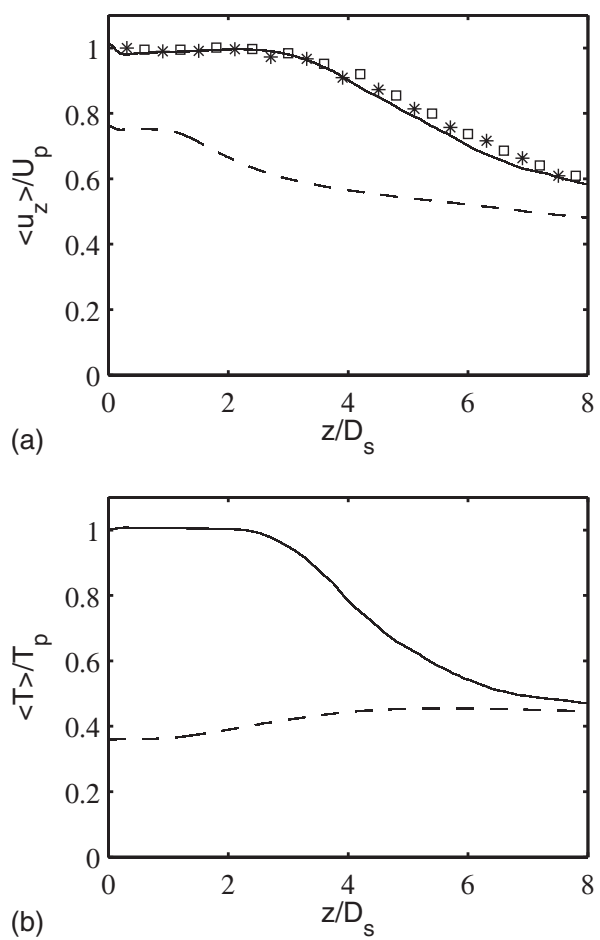

FIG. 6. Profiles of (a) mean axial velocity $\left\langle u_{z}\right\rangle / U_{p}$, (b) mean temperature $\langle T\rangle / T_{p}$; (-) along the jet centerline at $r=0$ and (- - ) along the secondary jet core centerline at $r=0.375 D_{s}$. CoJeN PIV measurements made along the jet centerline by: ( $\square$ ) Universidad Carlos III de Madrid, (*) University of Warwick, shifted axially by $-2 D_{s}$ for the comparison.

locity obtained along $r=0.375 D_{s}$ is also presented in the figure to display the velocity decay after the secondary potential core.

As final features of the jet mean flow, the profiles of mean temperature along $r=0$ and $r=0.375 D_{s}$ are shown in Fig. 6(b). The evolution toward the ambient temperature $T_{\mathrm{amb}} / T_{p}=0.37$ is quite rapid downstream of the jet primary core, whereas the mean static temperature slightly increases after the end of the secondary core. Unfortunately no experimental data are available for the comparison.

\section{Turbulent flow fields}

The rms levels of the fluctuating axial velocity are represented in Fig. 7. The higher levels, that are $\left[u_{z}^{\prime}\right]_{\mathrm{rms}}$ $\simeq 0.15 U_{p}$, are found in the early stage of development of the outer shear layer, whereas low levels are observed in the inner mixing layer between the two jet streams. Another re-

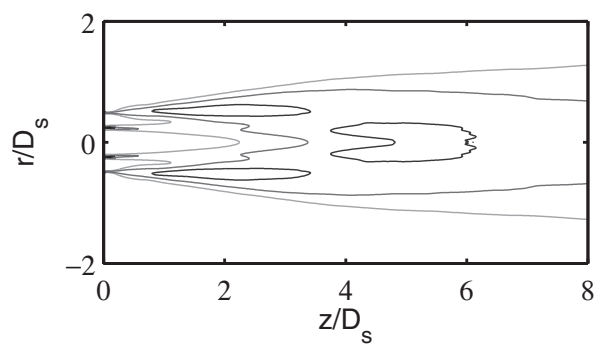

FIG. 7. Representation in the $(z, r)$ section: contours of rms fluctuating axial velocity $\left[u_{z}^{\prime}\right]_{\mathrm{rms}} / U_{p}=[0.045,0.09,0.135]$, from gray to black. 

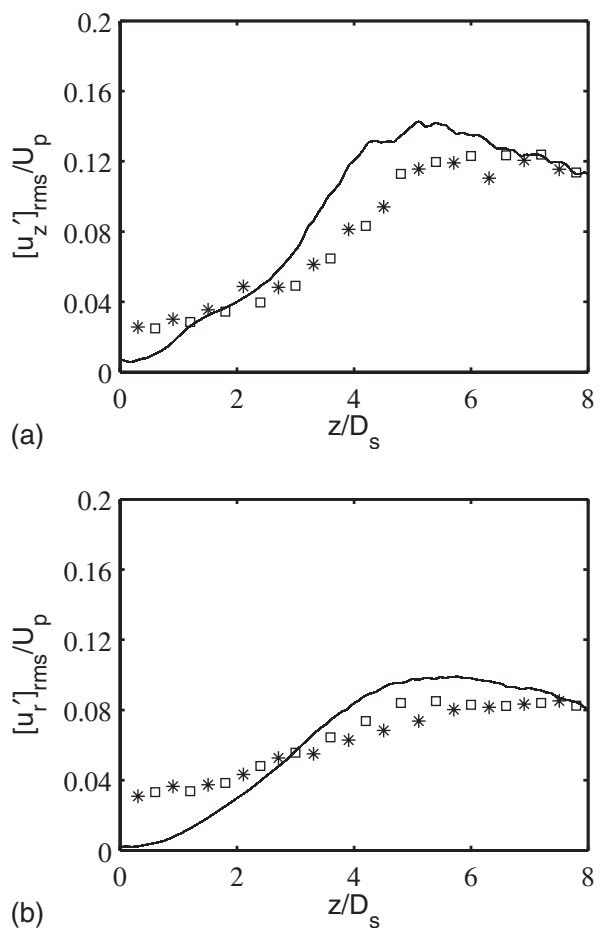

FIG. 8. Profiles along the jet centerline at $r=0$ of rms levels of (a) the fluctuating axial velocity $\left[u_{z}^{\prime}\right]_{\mathrm{rms}} / U_{p}$ and (b) the fluctuating radial velocity $\left[u_{r}^{\prime}\right]_{\mathrm{rms}} / U_{p}$. CoJeN PIV data by: ( $\square$ ) Universidad Carlos III de Madrid, (*) University of Warwick, shifted axially by $-2 D_{s}$.

gions exhibiting significant values of rms levels are also evidenced downstream. High rms values are indeed visible in the zones where the inner and outer mixing layers merge and on the centerline after the end of the primary potential core.

The centerline profiles of $\left[u_{z}^{\prime}\right]_{\mathrm{rms}}$ and $\left[u_{r}^{\prime}\right]_{\mathrm{rms}}$ are depicted in Fig. 8. They are compared with CoJeN PIV measurements shifted by $-2 D_{s}$ in the axial direction to match the different core lengths as previously in Fig. 6. The overall agreement between numerical and experimental curves is fair. The centerline turbulence levels are, however, initially lower in the LES than in the experiment, which emphasizes the differences in initial conditions. The peaks are also reached more rapidly in the LES, closer to the end of the potential core located at $z=3.5 D_{s}$. The peak levels provided by the LES, that are $\left[u_{z}^{\prime}\right]_{\mathrm{rms}} \simeq 0.14 U_{p}$ and $\left[u_{r}^{\prime}\right]_{\mathrm{rms}} \simeq 0.10 U_{p}$, are also slightly overestimated with respect to the experimental values $\left[u_{z}^{\prime}\right]_{\mathrm{rms}} \simeq 0.12 U_{p}$ and $\left[u_{r}^{\prime}\right]_{\mathrm{rms}} \simeq 0.08 U_{p}$.

\section{Turbulence development in the mixing layers}

A snapshot of the azimuthal vorticity field downstream of the nozzle lips is shown in Fig. 9. As mentioned in Sec. III A, a Von Kármán street (white color corresponding to positive vorticity and black color to negative vorticity) is observed in the inner shear layer between the primary hot stream and the secondary cold stream, which is in agreement with PIV measurements performed in the corresponding CoJeN jet. ${ }^{31}$ This behavior should be due to the small difference between velocities $U_{p}$ and $U_{s}$. This Von Kármán alley also seems to be persistent and displays several counterrotating vortex couples as in the CoJeN experiment.

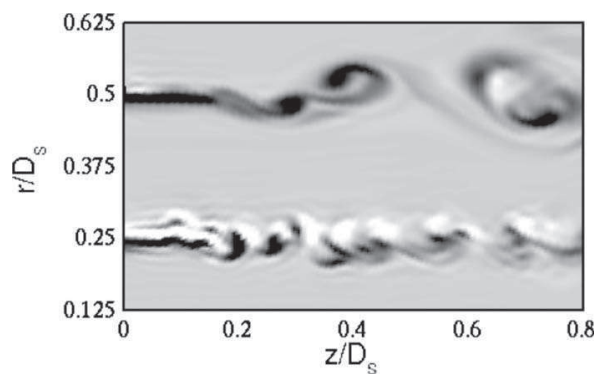

FIG. 9. Snapshot in the $(z, r)$ section of the azimuthal vorticity $\omega_{\phi}$ just downstream of the nozzle lips. The gray scale is defined for $-21.9 \leq \omega_{\phi} D_{s} / U_{p} \leq 7.3$ from dark to light.

To characterize the Von Kármán alley, velocity spectra are calculated along the inner lip line at $r=D_{s} / 4$. The spectrum of the fluctuating radial velocity obtained at $z=0.2 D_{s}$ is presented in Fig. 10(a). It is dominated by peaks with a fundamental peak at Strouhal number $\mathrm{Sr}_{\mathrm{VK}}=f D_{s} / U_{p}=8.8$. This peak frequency is the frequency of vortex shedding behind the inner nozzle lip. Based on the convection velocity $u_{c}$ $=0.56 U_{p}$ and the distance between vortices $d_{\mathrm{VK}}=0.064 D_{s}$, calculated at $z=0.2 D_{s}$ from axial correlations of $u_{r}^{\prime}$ velocity, a Strouhal number $\operatorname{Sr}_{\mathrm{VK}}=u_{c} D_{s} /\left(U_{p} d_{\mathrm{VK}}\right)=8.75$ is indeed obtained. For comparison, note also that a Strouhal number $\mathrm{Sr}_{\mathrm{VK}} \simeq 30$ has been measured in the CoJeN jet, the difference being certainly due to thinner experimental boundary layers.

In the spectrum of Fig. 10(a), owing to nonlinearity effects, subharmonic frequencies are also visible at Strouhal numbers $\mathrm{Sr}_{\mathrm{VK}} \times i / 8$ (that are $\mathrm{Sr}=1.1,2.2,3.3$, etc.). Because
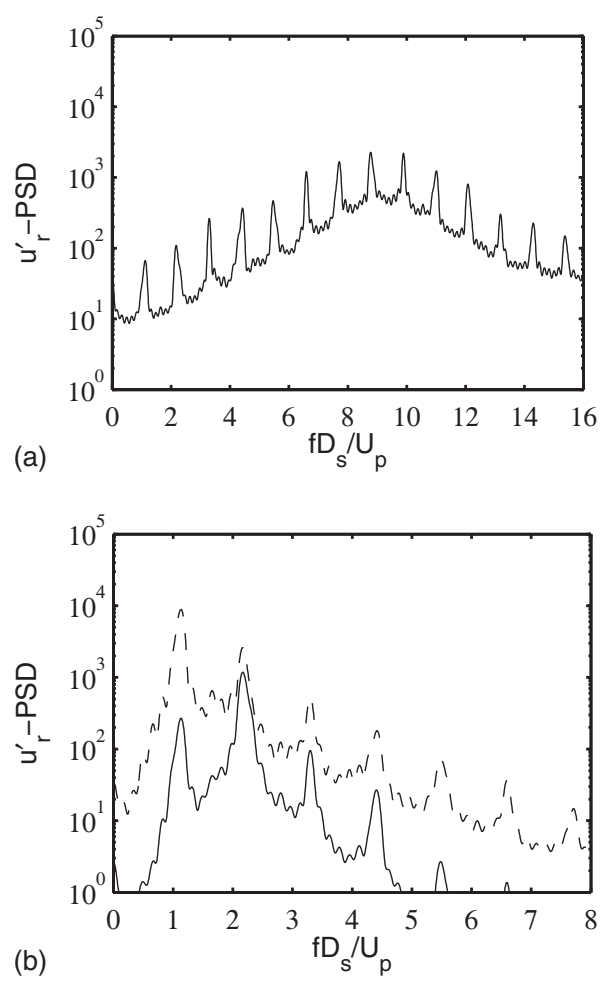

FIG. 10. Spectrum of the fluctuating radial velocity $u_{r}^{\prime}$ obtained as a function of Strouhal number $f D_{s} / U_{p}$ : (a) in the inner mixing layer at $r=0.25 D_{s}$ and $z=0.1 D_{s}$, (b) in the outer mixing layer at $r=0.5 D_{s}$ and (-) $z=0.1 D_{s} ;(--) z=0.4 D_{s}$. 
vortex shedding is an efficient noise generation mechanism, the development of the inner mixing layer in the LES might result in significant contributions to the sound field at these frequencies. This possibility is also supported by CoJeN measurements, ${ }^{31}$ which show acoustic tones compatible with the vortex street observed downstream of the inner nozzle lip.

Regarding the outer mixing layer at $r=D_{s} / 2$, it appears to develop in Fig. 9 in an organized fashion despite the high turbulence levels specified initially. Coherent vortices are generated in the shear layer, and visibly merge by pairing. The presence of vortex pairings is also supported by the spectra of velocity $u_{r}^{\prime}$ computed at $r=D_{s} / 2$. In Fig. 10(b), the spectrum calculated at $z=0.1 D_{s}$ is indeed dominated by a peak at $\mathrm{Sr}=2.2$, whereas at $z=0.4 D_{s}$ the subharmonic frequency at $\mathrm{Sr}=1.1$ is predominant. These frequencies are subharmonics of the Von Kármán fundamental frequency, which suggests that there is a coupling between the two mixing layers. Most likely acoustic waves radiated by the inner mixing layer force the outer shear layer. Finally, since vortex pairings are strong sound sources, the development of the outer shear layer might result in high noise components at the pairing subharmonics, in the same way as in twodimensional mixing layers, ${ }^{51,52}$ for instance.

\section{E. Turbulence properties along the potential core axes}

Some signals of flow quantities recorded along the primary and secondary potential core axes will be correlated with far-field pressure in Sec. V. The properties of these signals are examined in the present section. In order to track the dominant turbulent mechanisms along the two core axes, the variations of the spectral characteristics of the fluctuating radial velocity are first considered. The peak Strouhal numbers obtained along the lines at $r=0$ and $r=0.375 D_{s}$ are presented in Fig. 11(a). Both curves display large discontinuous steps.

Along the centerline at $r=0$, two steps characterized by $\mathrm{Sr}_{\text {peak }}=1.1$ then by $\mathrm{Sr}_{\text {peak }}=0.55$ are initially noticed for $z \leq D_{s}$. These two Strouhal numbers correspond to harmonics of the frequencies dominating in the mixing layers. Downstream, the peak Strouhal numbers take values around $\mathrm{Sr}_{\text {peak }}=0.32$ and finally around $\mathrm{Sr}_{\text {peak }}=0.16$ for $z \geq 3.2 D_{s}$. Quite obviously strong turbulent mechanisms occur along the primary core periodically at these two frequencies. At the end of the potential core, in particular, the spectral peak is obtained for $\mathrm{Sr}=0.16$. This result is illustrated in the corresponding velocity spectrum presented in Fig. 11(b), in which the component at $\mathrm{Sr}=0.32$ can also be seen.

Along the secondary core at $r=0.375 D_{s}$, the velocity spectra are successively dominated by the Strouhal numbers observed on the jet centerline. However, as shown in Fig. 11(a), the step at $\mathrm{Sr}_{\text {peak }}=0.55$ now lies from $z=D_{s}$ to $z=2.3 D_{s}$. The velocity spectrum at the end of the secondary potential core consequently reaches its maximum at $\mathrm{Sr}=0.55$ in Fig. 11(b). In this spectrum a component at $\mathrm{Sr}=0.32$ is also noticeable. Further downstream, the peak frequencies are very similar to those obtained at $r=0$, which
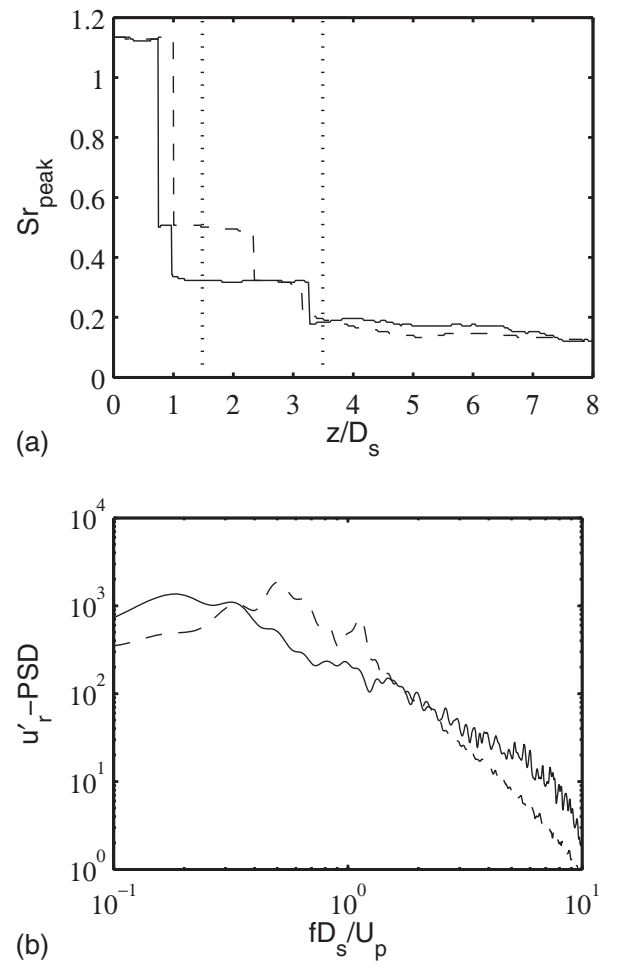

FIG. 11. Spectral properties of the fluctuating radial velocity $u_{r}^{\prime}$ along the potential core axes: (a) variations of the Strouhal number peak, $(-)$ at $r=0,(--)$ at $r=0.375 D_{s}$; (b) spectra at the end of (-) the inner core at $r=0$ and (- - ) the outer core at $r=0.375 D_{s}$, as a function of $S r=f D_{s} / U_{p}$.

indicates that the radial extent of the turbulent phenomena developing at the end of the primary core is significant.

To give additional information on turbulence, intermittency is analyzed along the two core axes as it has been previously done for single jets. ${ }^{9}$ A function $I(t)$ is defined arbitrarily from the vorticity component norm $\left|\omega_{r}\right|$ so that

$$
I(t)= \begin{cases}1 & \text { if }\left|\omega_{r}\right| \text { is lower than }\left\langle\left|\omega_{r}\right|\right\rangle / 2 \\ 0 & \text { otherwise, }\end{cases}
$$

and an intermittency factor is then evaluated as $\gamma=\langle I(t)\rangle$. This factor $\gamma$ is based here on the radial vorticity component $\omega_{r}=\partial u_{z} / \partial \phi-(1 / r) \partial u_{\phi} / \partial z$, and not on the full vorticity vector as in Ref. 9 because the other vorticity components are not available from the LES database. The norm $\left|\omega_{r}\right|$ is, however, a good indicator of the presence of three-dimensional vortical structures. Finally one can expect that $\gamma \simeq 0$ when the vorticity signal is fully laminar or turbulent, but that $\gamma$ becomes higher when the signal is intermittent.

The factor $\gamma$ calculated along the two core axes of the coaxial jet is represented in Fig. 12(a). Along the line at $r=0.375 D_{s}$, as $z$ increases, the value of $\gamma$ doubles to reach a peak one half-diameter before the end of the secondary potential core. At $r=0$, a similar behavior is noticed even if the variations are lower: the maximum of $\gamma$ is observed slightly upstream of the end of the inner core. Thus along both axes turbulence displays maximum intermittency just before the potential core end, as found in single jets. ${ }^{9}$

The variations in convection velocity $u_{c}$ along the potential core axes are also estimated using velocity cross correlations and plotted in Fig. 12(b). Significant variations are 

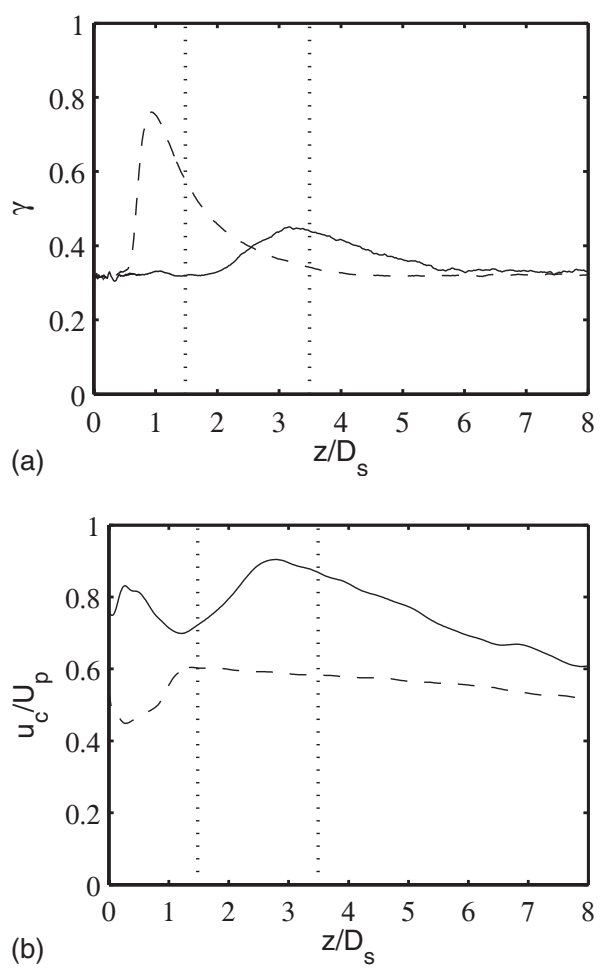

FIG. 12. Variations of (a) the intermittency factor $\gamma$ evaluated from vorticity and (b) the convection velocity $u_{c}$, along the potential core centerlines: $(-)$ at $r=0$ and (- - ) at $r=0.375 D_{s}$.

observed. The convection velocity especially increases in the potential cores to become, for instance, nearly $0.9 U_{p}$ on the centerline at $z=2.8 D_{s}$. The vortical structures traveling at this high convection speed are most probably accelerated from the shear layers, which likely generates noise. Note also that along the secondary core, both acceleration and deceleration probably take place, depending on whether vorticity is issued from the inner or the outer shear layer.

Some flow signals are finally shown at the maxima of intermittency found in Fig. 12(a), that are at $z=3 D_{s}$ on the centerline and at $z=D_{s}$ on the secondary core axis. Time evolutions of the axial velocity and radial vorticity norm obtained at the latter point for $\phi=0$, near the end of the secondary core, are first presented in Fig. 13. The velocity signal in Fig. 13(a) displays large deviations with respect to the inlet secondary velocity $U_{s}=0.75 U_{p}$. These deviations are both negative and positive, leading to variations between
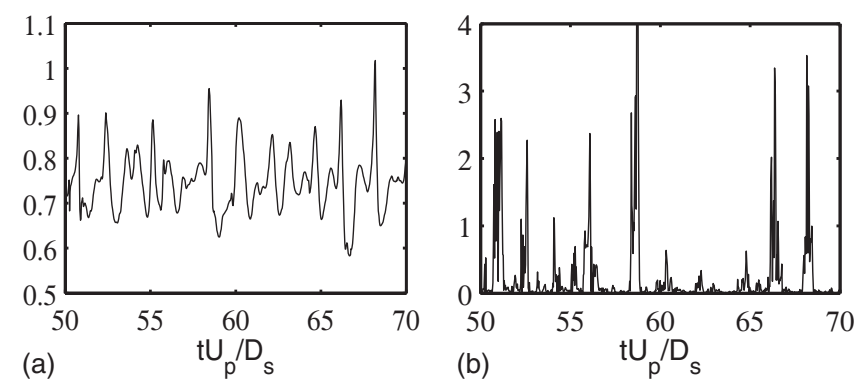

FIG. 13. Time evolution of (a) axial velocity $u_{z} / U_{p}$ and (b) radial vorticity norm $\left|\omega_{r}\right| D_{s} / U_{p}$ near the end of the secondary potential core, at $r=0.375 D_{s}, z=D_{s}$, and $\phi=0$.

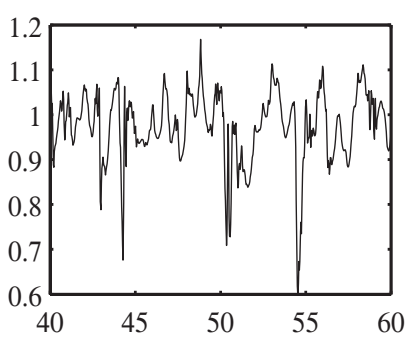

(a)

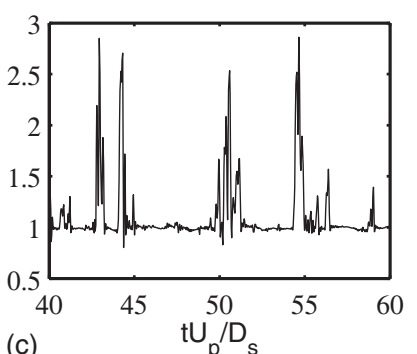

(c)

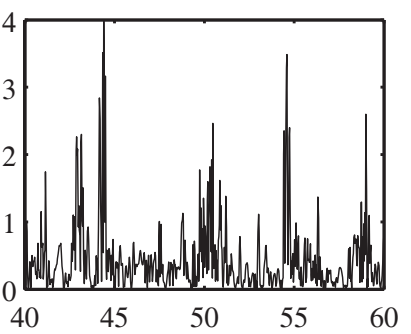

(b)

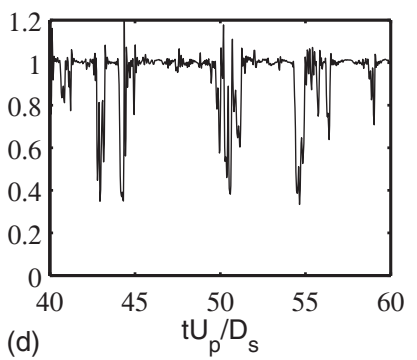

FIG. 14. Time evolution of (a) axial velocity $u_{z} / U_{p}$, (b) radial vorticity norm $\left|\omega_{r}\right| D_{s} / U_{p}$, (c) density $\rho / \rho_{p}$, and (d) temperature $T / T_{p}$ near the end of the primary potential core, at $r=0, z=3 D_{s}$, and $\phi=0$.

$0.6 U_{p}$ and $U_{p}$. A negative velocity deviation may correspond to the intrusion of the outer flow into the secondary core, whereas a positive deviation may be linked to the leak of the primary stream from the inner core. The velocity defects arise visibly periodically, at a Strouhal number $\mathrm{Sr} \simeq 0.5$ in agreement with the $\mathrm{Sr}_{\text {peak }}=0.55$ observed in Fig. 11. These turbulent phenomena also occur intermittently as illustrated by the vorticity norm in Fig. 13(b), which exhibits isolated peaks when velocity collapses in Fig. 13(a). This indicates the intrusion of vorticity from the outer mixing layer.

Signals recorded near the end of the primary potential core, at $r=0, z=3 D_{s}$, and $\phi=0$, are now represented in Fig. 14. In this hot jet stream, axial velocity, radial vorticity norm, as well as density and temperature are considered. The two former signals in Figs. 14(a) and 14(b) show that intermittently the flow decelerates down to $u_{z} \simeq 0.6 U_{p}$, while vorticity appears in the jet core. In the same time in Figs. 14(c) and 14(d), the density significantly increases while the temperature decreases down to $0.4 T_{p}$, that is close to the ambient temperature. These results give a clear evidence of the introduction of vortical structures developing in the cold outer shear layers into the hot primary core. This mechanism, similar to that found in single-stream jets, ${ }^{9}$ is likely a major sound source in the coaxial jet. The associated Strouhal number seems around $\mathrm{Sr}=0.2$, which is close to the peak value $\mathrm{Sr}_{\text {peak }} \simeq 0.16$ noticed in the velocity spectra at the end of the inner potential core in Fig. 11.

\section{ACOUSTIC RESULTS}

The near acoustic field determined directly by LES and the far acoustic field calculated using the extrapolation method are presented in this section and compared with CoJeN experimental data. 

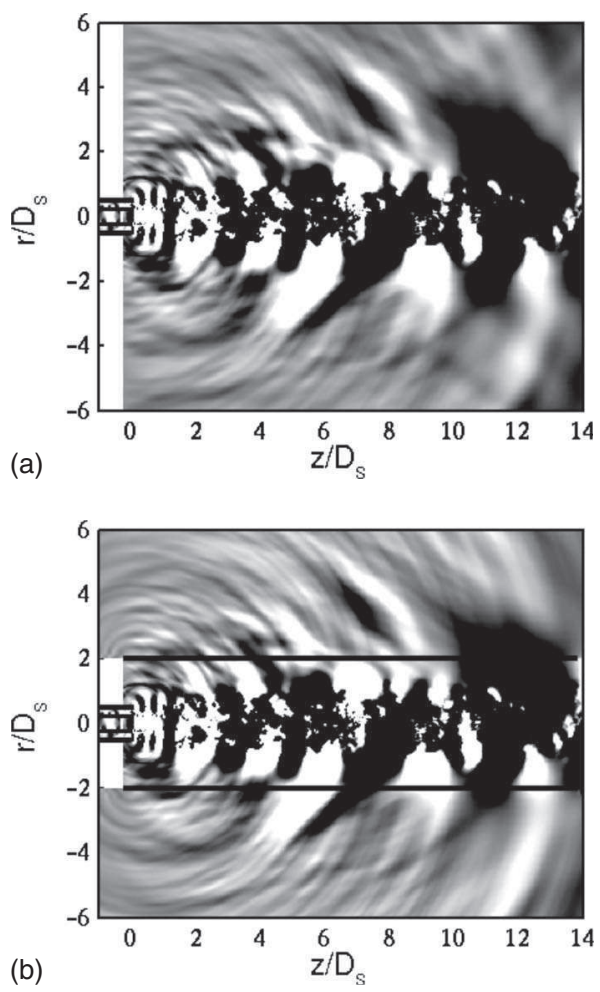

FIG. 15. Snapshots in the $(z, r)$ section of pressure fields obtained at LES time step 320 000: (a) LES pressure; (b) LES pressure in the central region, and, in peripheral regions, pressure computed from the acoustic equations using the control surface at $r=2 D_{s}$ indicated by black lines. The gray scale is defined for levels between -130 and $+130 \mathrm{~Pa}$, from light to dark.

\section{A. Pressure snapshots}

Snapshots of the near-pressure field obtained at time step 320000 for $r \leq 6 D_{s}$ are represented in Fig. 15. The pressure field provided directly by LES is displayed in Fig. 15(a), whereas the pressure field computed by solving the linear acoustical equations using the control surface at $r=2 D_{s}$ is given in Fig. 15(b). The coupling between the LES and the acoustical calculation seems to occur in an appropriate way. This is suggested by the continuity of the pressure field on either side of the interfaces, indicated by black lines in Fig. 15(b). There is a fair agreement with the near field determined by the LES, even if contributions of aerodynamic pressure $^{53,54}$ can be high in the vicinity of the jet flow in the LES. Note that the influence of the control surface was investigated in detail in a previous paper, ${ }^{50}$ where the acoustic extrapolation was also performed from surfaces at $3 D_{s}$ and $4 D_{s}$ from the jet axis. The results obtained with the surface at $r=2 D_{s}$ appeared to be the most accurate. The use of this surface located at $r=2 D_{s}$ and extending up to $z=13.8 D_{s}$ allows us, in particular, to minimize the possible effects due to the fact that the control surface is open.

A snapshot of the radiated pressure calculated from the linear acoustic equations is shown in Fig. 16. The large scale disparities between the sound waves and the turbulent region are clearly illustrated. Some changes in the noise features as the emission angle varies can also be noted. A

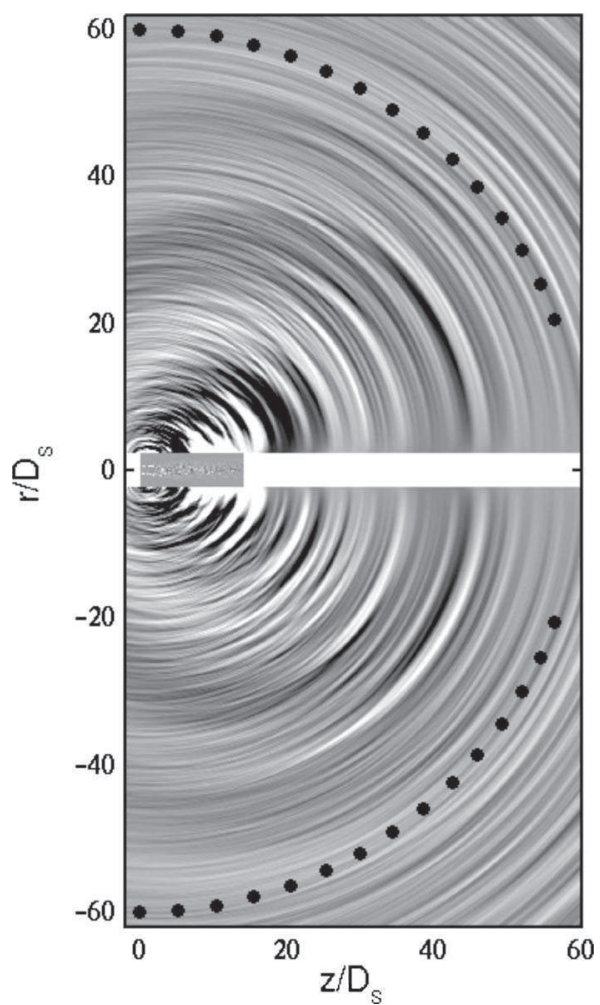

FIG. 16. Snapshots of pressure obtained at LES time step 320000 by solving the acoustic equations and of the LES vorticity norm in the central region. The gray scale is defined for pressure levels between -40 and +40 Pa, from light to dark. The black points indicate the observation points.

low-frequency noise characterized by a Strouhal number $\mathrm{Sr}=f D_{s} / U_{p} \simeq 0.05$ is moreover observed, in particular, in the sideline and upstream directions. This could unfortunately be parasitic waves generated at the LES outflow boundary. As a result, in order not to take into account the nonphysical acoustic components, the sound pressure levels will be computed only for $\mathrm{Sr} \geq 0.1$ in what follows. The observation points are finally represented in the figure. They are located at $60 D_{s}$ from the nozzle exit, for angles between $20^{\circ}$ and $90^{\circ}$ with respect to the jet direction.

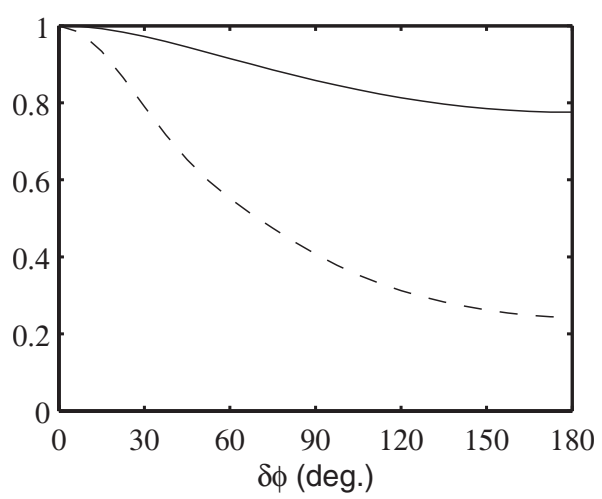

FIG. 17. Azimuthal cross-correlation functions of the fluctuating pressure obtained at $60 D_{s}$ from the nozzle exit, for radiation angles of (-) $30^{\circ}$ and (- - ) $60^{\circ}$. 

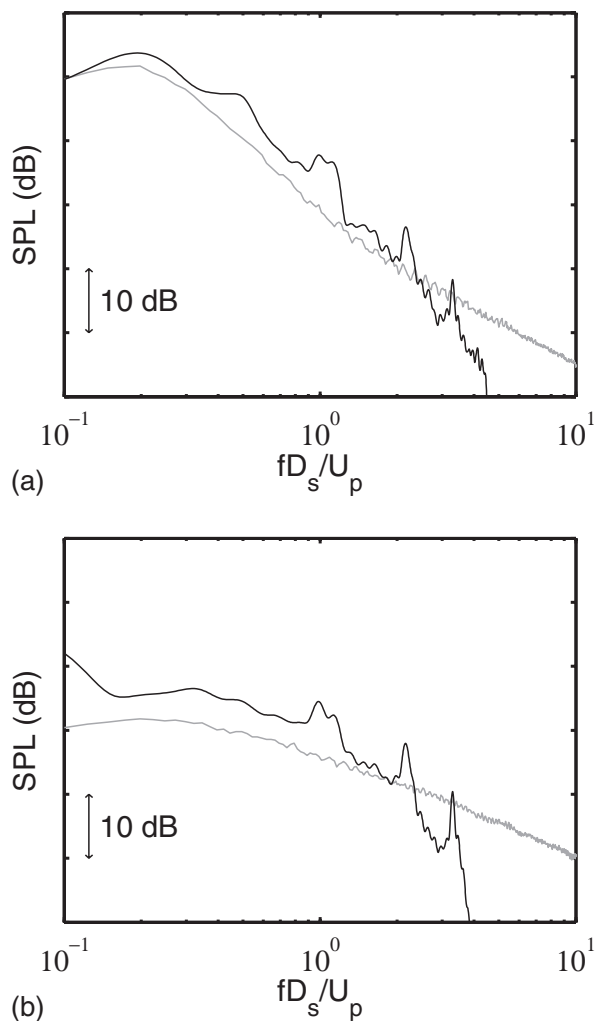

FIG. 18. Pressure spectra obtained at $60 D_{s}$ from the nozzle exit: (-) simulation results and (gray - ) CoJeN experimental data, for radiation angles relative to the jet direction of (a) $30^{\circ}$ and (b) $90^{\circ}$, as functions of Strouhal number $\mathrm{Sr}=f D_{s} / U_{p}$.

\section{B. Azimuthal cross correlations}

The spatial structure of the jet far-field noise is examined from the azimuthal correlations of the fluctuating far-field pressure. The correlation functions calculated at $30^{\circ}$ and $60^{\circ}$ relative to the jet from the broadband signals are shown in Fig. 17. The correlation levels are very high at the radiation angle of $30^{\circ}$, for which the correlation is even nearly 0.8 for an azimuthal separation angle of $180^{\circ}$. At the emission angle of $60^{\circ}$, the correlations are still significant but they are appreciably lower. A similar decrease with the radiation angle is observed in single-stream jets, ${ }^{8,55,56}$ and this behavior has been associated with jet noise components and sources. The present curves thus provide a first insight into noise generation.

\section{Sound pressure spectra}

The narrow-band sound pressure spectra calculated at the radiation angles of $30^{\circ}$ and $90^{\circ}$ are plotted in Fig. 18 and compared with CoJeN experimental data. Some peaks are visible in the spectra, especially at Strouhal numbers of 1.1, 2.2, 3.3, and 4.4. These frequencies correspond to the harmonic frequencies that are observed in velocity spectra obtained in the inner and outer mixing layers reported in Fig. 10. In the same way as it was assumed from CoJeN experimental results, ${ }^{31}$ these tones may be generated by the development of the Von Kármán vortex street exhibited in Fig. 9. The tones also likely result from vortex pairings in the outer shear layer. More precisely, in agreement with the nu-

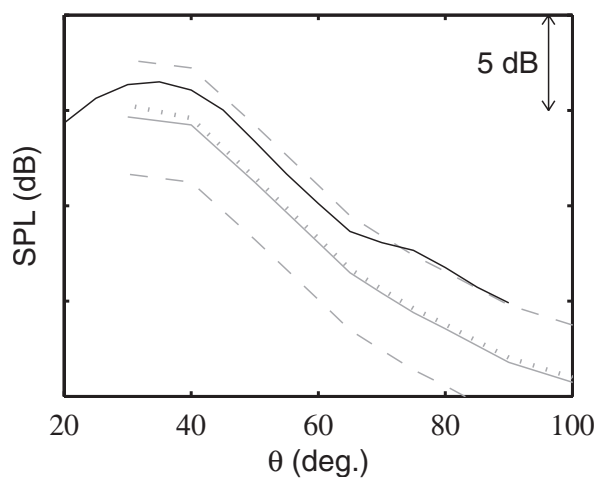

FIG. 19. Sound pressure levels obtained at $60 D_{s}$ from the nozzle exit, as a function of the radiation angle relative to the jet direction. Levels calculated for Strouhal numbers $\mathrm{Sr}=f D_{s} / U_{p} \geq 0.1$ : (-) simulation results, (gray -) CoJeN experimental data, (gray - - -) experimental data $\pm 3 \mathrm{~dB}$. Overall levels calculated for $\mathrm{Sr} \geq 0$ : (gray $\cdots \cdot \cdot)$ ) experimental data.

merical results obtained by Colonius et al. ${ }^{51}$ for twodimensional mixing layers, the contributions of pairings to noise appear significant at the two first subharmonics of the shear-layer fundamental Strouhal number of 2.2. The contribution at the second subharmonic frequency, i.e., at Strouhal number of 0.55 , is, in particular, especially noticeable in the downstream direction for a radiation angle of $30^{\circ}$ in Fig. 18(a).

The numerical spectra, however, agree well with the experimental spectra both in terms of overall shape and levels, from $\mathrm{Sr}=0.1$ up to a Strouhal number between 2 and 3, the cutoff Strouhal number of the simulation being around $\mathrm{Sr}=4$. Besides the agreement is particularly good in Fig. 18(a) with the results at $30^{\circ}$. The changes in the sound spectra with the radiation angles are also well predicted by the simulation. The pressure spectra especially broaden as the radiation angle increases, which is similar to what is observed for single-stream jets. ${ }^{8,57-59}$ The results suggest that the turbulent noise sources in the present jet at a high Reynolds number are properly taken into account by the LES.

\section{Sound pressure levels}

The numerical and experimental sound pressure levels obtained at $60 D_{s}$ from the nozzle exit are presented in Fig. 19. The levels are calculated over the Strouhal number range $\mathrm{Sr} \geq 0.1$. They display highest values for angles around $30^{\circ}$ relative to the jet direction, as classically found for single-jet noise. ${ }^{8,57-59}$ The agreement between the simulation and the experiment is very satisfactory, with no more than 3 $\mathrm{dB}$ between the results whatever the radiation angle may be. The LES sound levels are, however, higher at any angle. The overestimation, which could be partially due to the presence of harmonic tones in the sound spectra, is by $1.5 \mathrm{~dB}$ at the predominant radiation angle of $30^{\circ}$, and it increases at larger angles. It can also be noted that the effects of filtering the sound levels for $\mathrm{Sr} \geq 0.1$ are weak. From the experimental data, there are indeed very small differences between the overall sound levels (gray dotted line) and the levels filtered for $\mathrm{Sr} \geq 0.1$ (gray solid line). 


\section{CORRELATIONS BETWEEN TURBULENT AND ACOUSTIC FIELDS}

Possible evidences of noise generation in the coaxial jet are tracked from the simulation data using a causality method based on flow-acoustics correlations, as it has been done for single-stream jets. . $^{9,33,34,60}$

\section{A. Methodology}

To identify links between the flow field and the acoustic waves, correlations are computed between flow and far-field pressure signals. Based on previous results for single jets, for which high correlations are calculated between turbulence at the end of the potential core and the downstream sound waves, we focus here on the flow fields obtained along the primary and secondary potential core axes and on the farfield pressure radiated at $30^{\circ}$ relative to the jet direction. Flow-noise correlations involving shear-layer turbulence are not shown because noise generation along the shear layers is dominated by tones, whose properties have been discussed in Sec. II B to be likely biased by the numerical modeling of the inflow conditions. Due to the complexity of the jet flow and possible artifacts introduced by the inflow conditions with respect to experiment, the aim here is not to conduct an exhaustive study of noise source in the coaxial jet, but to show similarities with findings in single jets. Such observations could, in particular, support noise modellings ${ }^{24,26,27}$ considering coaxial jets as combinations of several single jets. In addition correlations of acoustical fluctuations with density and temperature in the present hot coaxial jet are calculated, which will allow us to deal with the question of investigating jet noise generation from such flow variables.

The quantities considered along both jet axes are the fluctuating longitudinal velocity $u_{z}^{\prime}$ and the radial vorticity norm $\left|\omega_{r}\right|$, whereas the fluctuating density $\rho^{\prime}$, as in the works of Panda, ${ }^{60}$ and temperature $T^{\prime}$ are also taken into account on the jet centerline. They are correlated with the radiated fluctuating pressure $p^{\prime}$ to determine the correlation functions $C u_{z}^{\prime}-p^{\prime}, C\left|\omega_{r}\right|-p^{\prime}, C \rho^{\prime}-p^{\prime}$, and $C T^{\prime}-p^{\prime}$. The normalized correlations between velocity $u_{z}^{\prime}$ at point $\mathbf{x}_{1}$ and pressure $p^{\prime}$ at point $\mathbf{x}_{2}$ are, for instance, determined in the following way:

$$
C u_{z}^{\prime}-p^{\prime}\left(\mathbf{x}_{1}, \mathbf{x}_{2}, \tau\right)=\frac{\left\langle u_{z}^{\prime}\left(\mathbf{x}_{1}, t\right) p^{\prime}\left(\mathbf{x}_{2}, t+\tau\right)\right\rangle}{\left\langle u_{z}^{\prime 2}\left(\mathbf{x}_{1}, t\right)\right\rangle^{1 / 2}\left\langle p^{\prime 2}\left(\mathbf{x}_{2}, t\right)\right\rangle^{1 / 2}},
$$

where $\tau$ is the time delay between the two signals.

The flow signals used for the correlations are broadband. However, due to the presence of tones in the sound spectrum in Fig. 18(a), preliminary calculations based on the broadband pressure provided quite periodic spots of correlation. In what follows, the correlations are therefore computed using the low-pass filtered pressure obtained by removing the spectral components so that $\mathrm{Sr} \geq 0.4$. The bound of $\mathrm{Sr}=0.4$ is chosen because it is lower than the second subharmonic frequency $\mathrm{Sr} \simeq 0.55$ at which vortex pairings have been found to contribute to the far-field pressure at $30^{\circ}$ in Sec. IV C. The other significant tones are also observed at higher frequencies. In this way the harmonic tones originating from the
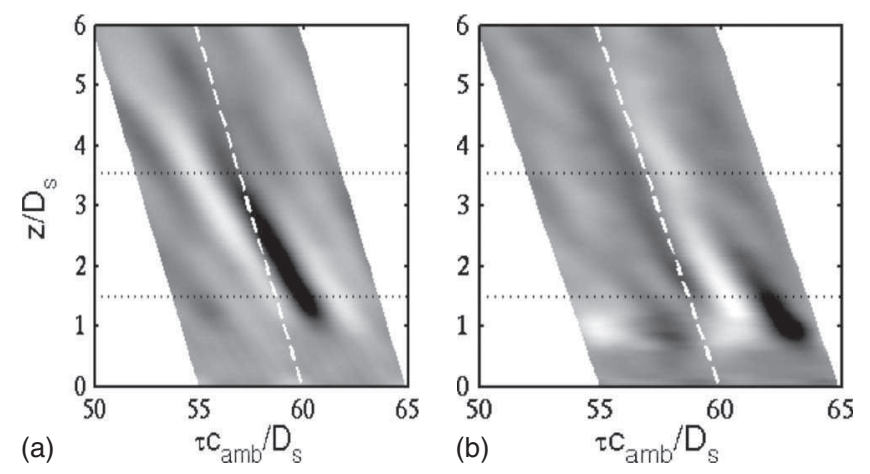

FIG. 20. Correlations between far-field low-pass filtered pressure $p^{\prime}$ at $30^{\circ}$ and (a) axial velocity $u_{z}^{\prime}$ and (b) radial vorticity norm $\left|\omega_{r}\right|$, along $r=0.375 D_{s}\left(X\right.$-axis: time delay $\tau c_{\text {amb }} / D_{s} ; Y$-axis: axial location $)$. The gray scales are defined from light to dark, (a) from -0.12 to 0.12 and (b) from -0.06 to 0.06 . The white line indicates the propagation time at the ambient sound speed and the dotted lines show the end of the two potential cores.

mixing layers should not contribute to the flow-noise correlations. The frequency range of $0 \leq \mathrm{Sr} \leq 0.4$ considered for the acoustic signal moreover contains the main components of the pressure spectrum in Fig. 18(a). The correlations calculated here may thus likely give some information on the generation of the dominant noise radiated in the downstream direction.

\section{B. Correlations with turbulence along the secondary potential core}

The normalized correlations between the low-pass filtered far-field pressure at $30^{\circ}$ and the fluctuating axial velocity and the radial vorticity norm along the secondary potential core axis are presented in Fig. 20. They are averaged in the azimuthal direction. Large regions of high correlation levels are visible on these space-time cartographies. The correlations are, in particular, especially significant around the end of the secondary potential core at $z=1.5 D_{s}$, for time delays slightly superior to the acoustic propagation time at the ambient speed of sound, indicated by a dashed line. At this position, they are dominated by negative values for $C u_{z}^{\prime}-p^{\prime}$, but positive values for $C\left|\omega_{r}\right|-p^{\prime}$.

Additional quantitative informations are given in Fig. 21 showing the correlation functions obtained from flow signals taken exactly at the end of the secondary potential core. Negative and positive extrema of correlations of $14 \%$ and

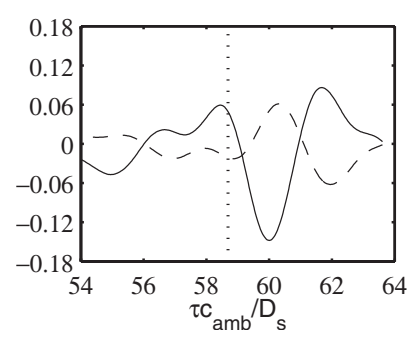

FIG. 21. Correlations between far-field low-pass filtered pressure at $30^{\circ}$ and turbulent quantities at $r=0.375 D_{s}$ at the end of the secondary potential core: (-) fluctuating axial velocity $\left(C u_{z}^{\prime}-p^{\prime}\right)$ and (- - ) radial vorticity norm $\left(C\left|\omega_{r}\right|-p^{\prime}\right)$. The dotted line represents the propagation time at the ambient sound speed. 


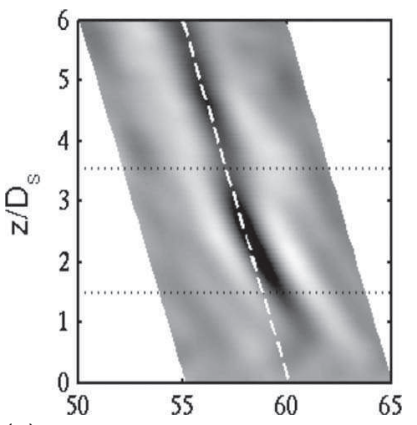

(a)

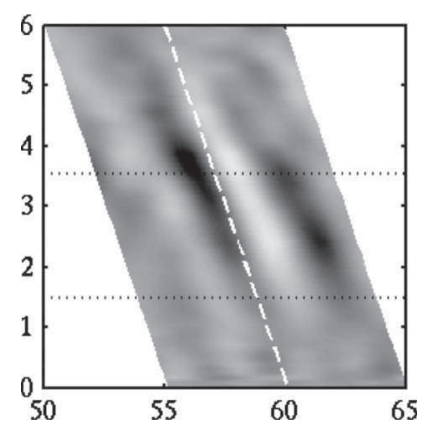

(b)
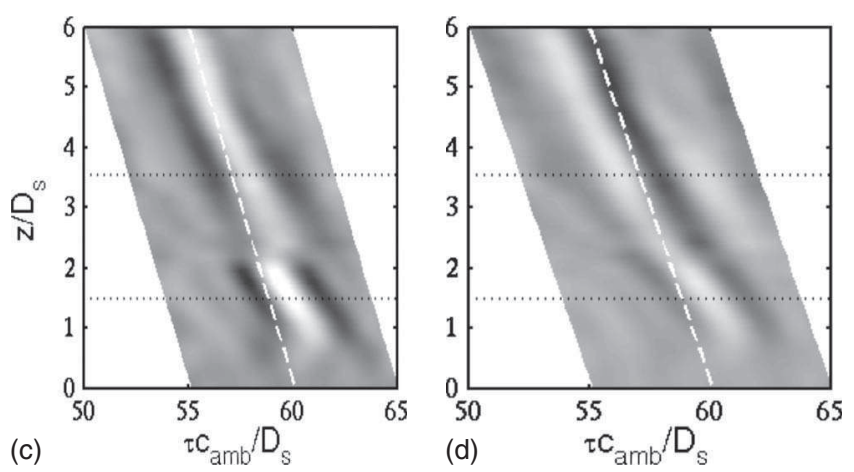

FIG. 22. Correlations between far-field low-pass filtered pressure $p^{\prime}$ at $30^{\circ}$ and (a) velocity $u_{z}^{\prime}$, (b) vorticity norm $\left|\omega_{r}\right|$, (c) density $\rho^{\prime}$, and (d) temperature $T^{\prime}$, along $r=0$ ( $X$-axis: time delay $\tau c_{\text {amb }} / D_{s} ; Y$-axis: axial location). The gray scales are defined $[(\mathrm{a}),(\mathrm{c})$, and (d)] from -0.28 to 0.28 and (b) from -0.12 to 0.12 . The white line indicates the propagation time at the ambient sound speed and the dotted lines show the end of the two potential cores.

$6 \%$ are observed, respectively, for the fluctuating velocity and the vorticity norm, just after the acoustic propagation time delay. As in single-stream jets, the velocity defects and vorticity outbursts occurring along the secondary potential core of the coaxial jet therefore seem to be correlated with the downstream noise. These results may indicate that the mechanisms described previously in Fig. 13 are connected with noise generation.

\section{Correlations with centerline turbulence}

The normalized correlations calculated between the lowpass filtered sound pressure and centerline flow signals are represented in Fig. 22 with the maps obtained for $C u_{z}^{\prime}-p^{\prime}$, $C\left|\omega_{r}\right|-p^{\prime}, C \rho^{\prime}-p^{\prime}$, and $C T^{\prime}-p^{\prime}$. Correlation levels between $10 \%$ and $20 \%$ are noticed. In Fig. 22(b), the far-field pressure is, in particular, seen to strongly correlate with the vorticity norm at the end of the primary potential core at $z$ $=3.5 D_{s}$, with high positive values reached around the acoustic propagation time. The correlation zones obtained from the fluctuating velocity in Fig. 22(a) are more extended, but significant negative correlation levels are also visible at the end of the primary core precisely at the acoustic propagation time. As in single jets, ${ }^{9,33}$ there are thus close links between the radiated downstream pressure waves and turbulence near the end of the core.

It is interesting to note that in a similar way density and temperature in this region correlate with sound pressure. They provide, respectively, spots of positive and negative
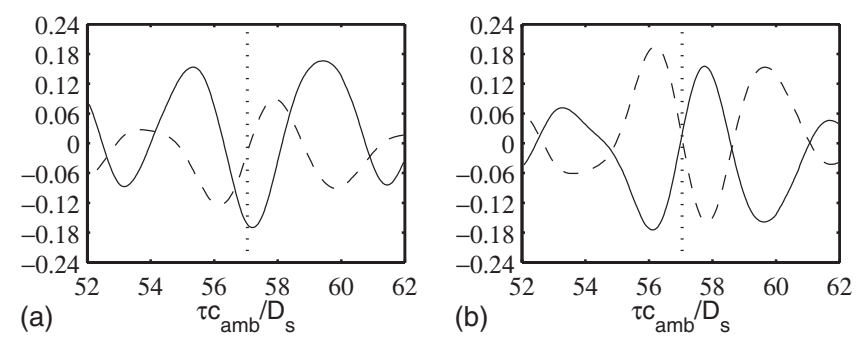

FIG. 23. Correlations between far-field low-pass filtered pressure at $30^{\circ}$ and turbulent quantities at $r=0$ at the end of the primary potential core: (a) (-) axial velocity $\left(C u_{z}^{\prime}-p^{\prime}\right)$ and (- - ) vorticity norm $\left(C\left|\omega_{r}\right|-p^{\prime}\right)$, (b) (-) density $\left(C \rho^{\prime}-p^{\prime}\right)$, and (- - ) temperature $\left(C T^{\prime}-p^{\prime}\right)$. The dotted line represents the propagation time at the ambient sound speed.

correlations in Figs. 22(c) and 22(d), which are well aligned with the spot of positive correlations from vorticity norm in Fig. 22(b). These results must be related to the coinciding deviations of vorticity norm, density, and temperature reported in the temporal signals of Fig. 14. In the heated jet stream, it thus appears possible to use density or temperature as indicators of the presence of vortical cold structure in the potential core. Significant correlation levels are also found further upstream, near the end of the secondary potential core at $z=1.5 D_{s}$. These correlations may, however, involve acoustic disturbances in the jet. The correlations from density and temperature are indeed here of the same sign, and correlations from vorticity norm in this region are negligible. In this case using density or temperature to evaluate flow-noise correlations might be misleading.

The shapes and magnitudes of the correlation functions obtained with the flow signals at the end of the primary core are finally illustrated in Fig. 23. Extremum correlation levels are around $16 \%$ for $C u_{z}^{\prime}-p^{\prime}, C \rho^{\prime}-p^{\prime}$, and $C T^{\prime}-p^{\prime}$, but $8 \%$ for $C\left|\omega_{r}\right|-p^{\prime}$. The extrema are in addition reached just at the acoustic propagation time delay for the axial velocity and very slightly after for vorticity, density, and temperature. This strongly supports direct connections with noise generation.

\section{CONCLUSION}

This paper presents the LES of a coaxial jet defined by the same stream velocities and temperatures and diameter ratio, as a jet studied in the EU project CoJeN, which provided both aerodynamic and acoustic experimental data. With the aim of computing directly practical jet noise, this simulation is a step forward because the flow configuration characterized by a coaxial geometry, a heated primary stream, and a high Reynolds number is complex. The jet considered is also more realistic regarding aeronautic applications. Very satisfactory results are obtained for the acoustic far-field directly obtained from the LES, without resorting to acoustical modeling. Sound pressure spectra and levels compare indeed well with CoJeN measurements, at any radiation angle. Some discrepancies attributed to numerical or physical artifacts, associated, for instance, with the outflow conditions or with the development of the mixing layers due to 
the modeling of the incoming boundary layers, are also identified in order to improve noise prediction in future simulations.

The simulation generated a database both for the turbulent flow and for the sound field of the coaxial jet. Thus for the first time to our knowledge, properties of the flow are described in detail and flow-noise correlations are computed to study noise generation mechanisms in a coaxial jet. The results are similar to those obtained for single-stream jets. High correlation levels are indeed observed between the downstream sound pressure and the turbulence around the end of the two potential cores. In these regions, the flow moreover exhibits intermittency, dominant Strouhal numbers persisting over large axial extents, and significant variations of the convection velocity. Noise generation therefore appears to be linked with the intrusion of vortical structures into the potential cores. Finally, the use of density or temperature instead of velocity or vorticity to compute flownoise correlations seems appropriate in the heated jet stream, but it might also indicate correlations with acoustic disturbances in the potential core.

\section{ACKNOWLEDGMENTS}

This work was supported by the EU Sixth Framework Project CoJeN (Computation of Coaxial Jet Noise, EC Contract No. AST3-CT-2003-502790), as well as by the ANR (the French National Research Agency) Program No. NT054_42264 "Identification des mécanismes générateurs de bruit dans les écoulements libres." The authors thank François Coiffet for processing data. They gratefully acknowledge the Institut du Développement et des Ressources en Informatique Scientifique (IDRIS-CNRS) for providing computing time and for its technical assistance. A part of the computations was also carried out on the supercomputers of the CEA (the French Atomic Energy Agency).

${ }^{1}$ C. K. W. Tam, "Jet noise: since 1952," Theor. Comput. Fluid Dyn. 10, 393 (1998).

${ }^{2}$ C. K. W. Tam, "Supersonic jet noise," Annu. Rev. Fluid Mech. 27, 17 (1995).

${ }^{3}$ T. Colonius and S. K. Lele, "Computational aeroacoustics: progress on nonlinear problems of sound generation," Prog. Aerosp. Sci. 40, 345 (2004).

${ }^{4}$ M. Wang, J. B. Freund, and S. K. Lele, "Computational prediction of flow-generated sound," Annu. Rev. Fluid Mech. 38, 483 (2006).

${ }^{5}$ J. B. Freund, "Noise sources in a low-Reynolds-number turbulent jet at Mach 0.9," J. Fluid Mech. 438, 277 (2001).

${ }^{6}$ C. Bogey, C. Bailly, and D. Juvé, "Noise investigation of a high subsonic, moderate Reynolds number jet using a compressible LES," Theor. Comput. Fluid Dyn. 16, 273 (2003).

${ }^{7}$ C. Bailly and C. Bogey, "Contributions of CAA to jet noise research and prediction,” Int. J. Comput. Fluid Dyn. 18, 481 (2004).

${ }^{8} \mathrm{C}$. Bogey and C. Bailly, "Investigation of downstream and sideline subsonic jet noise using large eddy simulations," Theor. Comput. Fluid Dyn. 20, 23 (2006).

${ }^{9}$ C. Bogey and C. Bailly, "An analysis of the correlations between the turbulent flow and the sound pressure fields of subsonic jets," J. Fluid Mech. 583, 71 (2007).

${ }^{10}$ C. Bogey, S. Barré, and C. Bailly, "Direct computation of the noise generated by subsonic jets originating from a straight pipe nozzle," Int. J. Aeroacoust. 7, 1 (2008).

${ }^{11}$ A. Uzun and M. Hussaini, "Investigation of high frequency noise generation in the near-nozzle region of a jet using large eddy simulation," Theor. Comput. Fluid Dyn. 21, 291 (2007).
${ }^{12}$ I. M. A. Al-Qadi and J. N. Scott, "High-order three-dimensional numerical simulation of a supersonic rectangular jet," AIAA Paper No. 2003-3237, 2003.

${ }^{13}$ J. Berland, C. Bogey, and C. Bailly, "Numerical study of screech generation in a planar supersonic jet," Phys. Fluids 19, 075105 (2007).

${ }^{14}$ X. D. Li and J. H. Gao, "Numerical simulation of the three-dimensional screech phenomenon from a circular jet," Phys. Fluids 20, 035101 (2008).

${ }^{15} \mathrm{U}$. Paliath and P. J. Morris, "Prediction of jet noise from circular beveled nozzles," AIAA Paper No. 2005-3096, 2005.

${ }^{16}$ K. Viswanathan, M. L. Shur, P. R. Spalart, and M. Kh. Strelets, "Computation of the flow and noise of round and beveled nozzles," AIAA Paper No. 2006-2445, 2006.

${ }^{17}$ N. Andersson, L.-E. Eriksson, and L. Davidson, "LES prediction of flow and acoustic field of a coaxial jet," AIAA Paper No. 2005-2884, 2005.

${ }^{18}$ A. Vuillemin, P. Loheac, G. Rahier, F. Vuillot, and N. Lupoglazoff, "Aeroacoustic numerical method assessment for a double stream nozzle," AIAA Paper No. 2005-3043, 2005.

${ }^{19}$ M. Mihăescu, E. Gutmark, R.-Z. Szasz, and L. Fuchs, "Flow and acoustics of a coaxial nozzle: a sensitivity to the inlet boundary conditions," AIAA Paper No. 2006-1387, 2006.

${ }^{20}$ I. H. Tristanto, G. J. Page, and J. J. McGuirk, "Large eddy simulation of hot coaxial jets," AIAA Paper No. 2006-2497, 2006.

${ }^{21}$ D. Eschricht, J. Yan, U. Michel, and F. Thiele, "Prediction of jet noise from a coplanar nozzle," AIAA Paper No. 2008-2969, 2008.

${ }^{22}$ H. K. Tanna, "An experimental study of jet noise. Part I: Turbulent mixing noise,” J. Sound Vib. 50, 405 (1977).

${ }^{23}$ K. Viswanathan, "Aeroacoustics of hot jets," J. Fluid Mech. 516, 39 (2004).

${ }^{24}$ N. W. M. Ko and A. S. H. Kwan, "The initial region of subsonic coaxial jets," J. Fluid Mech. 73, 305 (1976).

${ }^{25}$ C. B. da Silva, G. Balarac, and O. Métais, "Transition in high velocity ratio coaxial jets analysed from direct numerical simulations," J. Turbul. 4, 024 (2003).

${ }^{26}$ M. J. Fisher, G. A. Preston, and C. J. Mead, "A modelling of the noise from simple coaxial jets, part I: With unheated primary flows," J. Sound Vib. 209, 385 (1998).

${ }^{27}$ M. J. Fisher, G. A. Preston, and C. J. Mead, "A modelling of the noise from simple coaxial jets, part II: With heated primary flows," J. Sound Vib. 209, 405 (1998).

${ }^{28}$ C. Tinney and P. Jordan, "The near pressure field of coaxial subsonic jets," J. Fluid Mech. 611, 175 (2008).

${ }^{29}$ C. Bogey and C. Bailly, "A family of low dispersive and low dissipative explicit schemes for flow and noise computations," J. Comput. Phys. 194, 194 (2004)

${ }^{30}$ F. Vuillot, N. Lupoglazoff, and G. Rahier, "Double-stream nozzles flow and noise computations and comparisons to experiments," AIAA Paper No. 2008-0009, 2008.

${ }^{31}$ J. Nogueira, M. Legrand, S. Nauri, P. A. Rodríguez, and A. Lecuona, "Analysis of the vortex street generated at the core-bypass lip of a jet engine nozzle," Top. Appl. Phys. 112, 419 (2008).

${ }^{32}$ A. J. Skeen, "The development of high-speed PIV techniques and their application to jet noise measurements," Ph.D thesis, University of Warwick, UK, 2006.

${ }^{33}$ M. Schaffar, "Direct measurements of the correlation between axial in-jet velocity fluctuations and far field noise near the axis of a cold jet," J. Sound Vib. 64, 73 (1979).

${ }^{34}$ J. Panda, R. G. Seasholtz, and K. A. Elam, "Investigation of noise sources in high-speed jets via correlation measurements," J. Fluid Mech. 537, 349 (2005).

${ }^{35}$ J. Berland, C. Bogey, O. Marsden, and C. Bailly, "High-order, low dispersive and low dissipative explicit schemes for multiple-scale and boundary problems," J. Comput. Phys. 224, 637 (2007).

${ }^{36}$ G. S. Constantinescu and S. K. Lele, "A highly accurate technique for the treatment of flow equations at the polar axis in cylindrical coordinates using series expansions," J. Comput. Phys. 183, 165 (2002).

${ }^{37}$ C. Bogey and C. Bailly, "Large eddy simulations of transitional round jets: influence of the Reynolds number on flow development and energy dissipation," Phys. Fluids 18, 065101 (2006).

${ }^{38}$ C. Bogey and C. Bailly, "Decrease of the effective Reynolds number with eddy-viscosity subgrid-scale modeling," AIAA J. 43, 437 (2005).

${ }^{39}$ C. Bogey and C. Bailly, "Large eddy simulations of round free jets using explicit filtering with/without dynamic Smagorinsky model," Int. J. Heat Fluid Flow 27, 603 (2006).

${ }^{40} \mathrm{C}$. Bogey and C. Bailly, "Three-dimensional non reflective boundary con- 
ditions for acoustic simulations: far-field formulation and validation test cases," Acta Acust. 88, 463 (2002).

${ }^{41}$ C. Bogey and C. Bailly, "Effects of inflow conditions and forcing on a Mach 0.9 jet and its radiated noise," AIAA J. 43, 1000 (2005).

${ }^{42}$ K. B. M. Q. Zaman, "Effect of the initial condition on subsonic jet noise," AIAA J. 23, 1370 (1985).

${ }^{43}$ J. E. Bridges and A. K. M. F. Hussain, "Roles of initial conditions and vortex pairing in jet noise," J. Sound Vib. 117, 289 (1987).

${ }^{44}$ K. Viswanathan and L. T. Clark, "Effect of nozzle internal contour on jet aeroacoustics," Int. J. Aeroacoust. 3, 103 (2004).

${ }^{45}$ D. J. Bodony and S. K. Lele, "On the current status of jet noise predictions using large-eddy simulation,” AIAA J. 46, 364 (2008).

${ }^{46}$ K. B. M. Q. Zaman, "Far-field noise of a subsonic jet under controlled excitation,” J. Fluid Mech. 152, 83 (1985).

${ }^{47}$ A. S. Lyrintzis, "Surface integral methods in computational aeroacoustics-From the (CFD) near-field to the (Acoustic) far-field," Int. J. Aeroacoust. 2, 95 (2003).

${ }^{48}$ X. Gloerfelt, C. Bailly, and D. Juvé, "Direct computation of the noise radiated by a subsonic cavity flow and application of integral methods," J. Sound Vib. 266, 119 (2003).

${ }^{49}$ J. B. Freund, "A simple method for computing far-field sound in aeroacoustic computations," J. Comput. Phys. 157, 796 (2000).

${ }^{50} \mathrm{C}$. Bogey, S. Barré, and C. Bailly, "Direct computation of the noise generated by a hot coaxial jet," AIAA Paper No. 2007-3587, 2007.
${ }^{51}$ T. Colonius, S. K. Lele, and P. Moin, "Sound generation in a mixing layer," J. Fluid Mech. 330, 375 (1997).

${ }^{52}$ C. Bogey, C. Bailly, and D. Juvé, "Numerical simulation of the sound generated by vortex pairing in a mixing layer," AIAA J. 38, 2210 (2000).

${ }^{53}$ R. E. A. Arndt, D. F. Long, and M. N. Glauser, "The proper orthogonal decomposition of pressure fluctuations surrounding a turbulent jet," J. Fluid Mech. 340, 1 (1997).

${ }^{54}$ F. Coiffet, P. Jordan, J. Delville, Y. Gervais, and F. Ricaud, "Coherent structures in subsonic jets: a quasi-irrotational source mechanism?” Int. J. Aeroacoust. 5, 67 (2006).

${ }^{55}$ L. Maestrello, "Two points correlations of sound pressure in the far field of a jet: Experiment," NASA Report No. NASA-TMX-72835 (1976).

${ }^{56}$ D. Juvé, M. Sunyach, and G. Comte-Bellot, "Filtered azimuthal correlations in the acoustic far field of a subsonic jet," AIAA J. 17, 112 (1979).

${ }^{57}$ E. Mollo-Christensen, M. A. Kolpin, and J. R. Martucelli, "Experiments on jet flows and jet noise far-field spectra and directivity patterns," J. Fluid Mech. 18, 285 (1964).

${ }^{58}$ P. A. Lush, "Measurements of subsonic jet noise and comparison with theory," J. Fluid Mech. 46, 477 (1971).

${ }^{59}$ K. K. Ahuja, "Correlation and prediction of jet noise," J. Sound Vib. 29, 155 (1973).

${ }^{60} \mathrm{~J}$. Panda, "Experimental investigation of turbulent density fluctuations and noise generation from heated jets," J. Fluid Mech. 591, 73 (2007). 